\title{
Implicaciones del tsunami de Tohoku del año 2011 para la gestión de desastres naturales en Japón
}

\author{
Implications of the 2011 Tohoku tsunami for risk management in Japan
}

Fecha de entrega: 22 de enero 2012

Fecha de aceptación: 2 de mayo 2012

\section{Tomoya Shibayama ${ }^{1}$, Miguel Esteban ${ }^{1}$, Ioan Nistor ${ }^{2}$, Hiroshi Takagi ${ }^{3}$, Thao Nguyen ${ }^{4}$, Ryo Matsumaru ${ }^{5}$, Takahito Mikami ${ }^{1}$, Koichiro Ohira ${ }^{1}$ y Akira Ohtani ${ }^{1}$}

${ }^{1}$ Department of Civil and Environmental Engineering, Waseda University, 3-4-1 Ookubo, Shinjuku-ku, Tokyo, T169-8555, Japan, shibayama@waseda.jp, esteban@aoni.waseda.jp,t.mikami@asagi.waseda.jp,chonchan.factory@gmail.com, r-ohtani@ruri.waseda.jp ${ }^{2}$ Department of Civil Engineering, University of Ottawa, 161 Louis Pasteur St., A115, Ottawa, Ontario K1N 6N5, Canada, inistor@uottawa.ca

${ }^{3}$ Department of International Development, Tokyo Institute of Technology, Ookayama 2-12-1-S 6-3, Meguro-ku, Tokyo, T152-8550, Japan takagi@ide.titech.ac.jp

${ }^{4}$ Department of Port and Coastal Engineering, Ho Chi Minh City University of Technology, 268 Ly Thuong Kiet Street, District 10, HCMC Vietnam, ndthao@hcmut.edu.vn

${ }^{5}$ IRM Ltd, Kasuya 2 chome, Setagaya-ku, 1570063, Tokyo Japan, matsumaru.ryo@irm-j.co.jp

\begin{abstract}
El 11 de Marzo del 2011 un terremoto de magnitud 9.0 en la escala Richter generó un devastador tsunami que arrasó una extensa área de la costa noreste de Japón. El Gran Terremoto y Tsunami del Este ha sido descrito como un evento que solo sucede una vez cada varios miles de años aproximadamente, y como consecuencia de éste es necesario que la filosofía sobre Gestión de Desastres Naturales en Japón sea revisada para tener en cuenta varias lecciones aprendidas a raíz de este desastre. En este artículo los auotres presentan los resultados de investigaciones de terreno en el área de Tohoku. Se detalla el daño en varias localidades y se analizan los mecanismos de daño sobre diversos tipos de estructuras. Los autores también presentan una serie de cambios que están ocurriendo en la filosofia de gestión de riesgos en Japón. Esencialmente, la idea que sólo los métodos de infraestructura física son siempre capaces de proteger las vidas de la población local ha sido descartada, y aunque se mantiene la idea de que estos métodos pueden resultar útiles para la protección de las propiedades, en última instancia los métodos de planificación deberían formar la pieza más importante del sistema de medidas para la protección de las vidas de las personas en estas áreas.
\end{abstract}

Palabras clave: tsunami, Tohoku, Gestión de Desastres Naturales
On March 11 th 2011, a large earthquake of magnitude 9.0 on the Richter scale occurred offshore the northeast coast of Japan, generating a major tsunami which devastated large parts of Japan's north-eastern coastline. The "Great Eastern Japan Earthquake and Tsunami" has been described as one in several thousand years event, and as a consequence it is necessary that the revising of the philosophy behind Coastal Disaster Management in Japan take into account the lessons learned from this latest disaster. In this article, the authors present the results of their field surveys in the Tohoku area, describing the damage in various places and analysing the failure mechanism of a variety of coastal structures. The authors also present some changes that are currently occurring in the risk management philosophy in Japan. Essentially, the idea that infrastructure measures can always protect against the loss of life has been discarded, and although they can play a part in the protection of property ultimately planification measures should be the cornerstone of the strategy to protect people's lives in coastal areas.

Keywords: tsunami, Tohoku, Disaster Risk Management

costa noreste de Japón, generando un tsunami que causó graves daños en las zonas costeras. Un gran número de edificios, incluso muchos de ellos construidos en hormigón armado, fueron destruidos o sufrieron daños extensos, y 
una gran parte de la flota pesquera terminó encallada en tierra. Muchas estructuras de defensa costera como diques, rompeolas $\mathrm{u}$ otras estructuras construidas para resistir tsunamis, también sufrieron graves daños. Ha sido uno de los peores tsunamis que ha afectado Japón a lo largo de su historia. De hecho, el así llamado Gran Terremoto y Tsunami del Este está considerado como un evento que se produce solo cada varios miles de años, al igual que el tsunami de Jogan del año 869 d.C. (Sawai et al., 2006). Esta zona de Japón, conocida con el nombre de Tohoku, ha sido frecuentemente atacada por tsunamis, como el Tsunami de Keicho en el año 1611, donde las olas llegaron hasta $4 \mathrm{~km}$ tierra adentro, causando grandes daños en la región (Sawai et al., 2006), o los tsunamis de Meiji-Sanriku de 1896 y Showa-Sanriku de 1933.

Como consecuencia de este tsunami se hace necesario revisar la filosofía detrás de la Gestión de Desastres Naturales en Japón. El desastre ha proporcionado muchas lecciones de cómo se podrían mejorar muchos aspectos de esta gestión, y estas lecciones afectarán las consideraciones teóricas, morales y éticas de dicha gestión. En este artículo los autores describen las principales características del evento y sus implicancias.

Mucho de lo que será discutido tiene su base en problemas intrínsecos a la actual manera de gestionar desastres naturales. Durante muchos años se ha debatido entre los Ingenieros de Costas japoneses y la comunidad que gestiona los desastres naturales si los métodos físicos de protección basados sólo en infraestructura, como los rompeolas o diques, son preferibles a los métodos de planificación como los sistemas de alerta o planes de evacuación. La magnitud del evento de marzo del 2011 ha intensificado el debate, y de hecho en Japón se está comenzando a llegar a un consenso a este respecto. La idea de que las estructuras de protección son siempre capaces de proteger la vida de los habitantes de la costa ha sido abandonada, especialmente a la luz de este último evento. La función de estas estructuras es por tanto dar protección a las propiedades durante los eventos de mayor frecuencia, pero de una intensidad menor, típicamente con un periodo de retorno de entre 50 a 60 años y entre 150 a160 años (intervalos de frecuencia usados en Japón). Estos eventos comienzan a ser referidos como "Tsunamis de Nivel 2". El objetivo de los métodos de planificación sería la protección de vidas, y estaría diseñado teniendo en mente los eventos más infrecuentes pero de mayor intensidad que comienzan a ser definidos como "Tsunamis de Nivel 1". El costo financiero de construir estructuras para proteger contra tsunamis es bastante elevado, y su efectividad no está del todo clara, sobre todo para eventos superiores al tsunami de diseño, como sucedió en marzo del 2011.

En el futuro la construcción de infraestructura sólo debería proceder si se establece que tienen sentido desde un punto de vista de costo-beneficio, especialmente considerando que sólo se espera que protejan las propiedades de las zonas costeras. Hoy en día no está claro hasta qué punto los métodos físicos contribuyeron a aliviar el daño causado por el tsunami, especialmente dado el extenso (a veces catastrófico) daño que sufrieron estas estructuras. Un análisis preliminar de la altura de ola en frente y detrás del rompeolas situado en la bahía de Kamaishi muestra como la estructura podría haber contribuido a reducir la altura de inundación entre un 40 y 50\% (utilizando datos del Tohoku Earthquake Tsunami Joint Survey Group (2011) y de PARI (2011)), aunque realmente es necesario investigar este fenómeno mucho más a fondo.

Una parte de esta discusión sobre los métodos de planificación y de infraestructura se centra en si las áreas costeras pueden ser consideradas como lugares de recreo o como el origen de posibles amenazas. ¿Se debería preservar la belleza de estas áreas, o protegerlas de potenciales ataques provenientes del mar? Japón es un país que periódicamente experimenta desastres naturales como terremotos, tsunamis y tifones, entre otros. Para protegerse contra tifones y tsunamis es necesario no solo construir defensas costeras sino que también es necesario la adecuación de ríos y laderas de montañas para proteger contra otras consecuencias de estos desastres, como inundaciones y remociones en masa. Por lo tanto, las consideraciones estéticas y de ingeniería pertinentes al caso de Japón no son necesariamente las mismas de otros países. Esto se refleja también en la mentalidad de la población e Ingenieros Civiles japoneses, que asocian la idea de problemas costeros con amenazas y como protegerse de ellas. Por ejemplo, la costa de Sanriku tiene una economía que depende de la pesca y otras industrias 
asociadas con esta actividad. Para la población que vive en estas áreas las consideraciones estéticas son seguramente secundarias comparado con la protección de vidas y de su estilo de vida. Por esto la implementación de métodos de defensa físicos es esencial para que la zona continúe siendo habitada y que la población viva en relativa paz con el mar. Este sentimiento en Japón es en general bastante diferente al de otros países de Europa o América del Norte, donde la implementación de estructuras de defensa sería difícil debido a la oposición de la población del lugar, que protestaría sobre el impacto de las estructuras sobre el ambiente.

El Gran Terremoto y Tsunami del Este del 2011 sin duda conllevará a profundas reflexiones sobre los conceptos y formas de gestión de riesgos referentes a tsunamis en Japón y en el mundo. La gran cantidad de daños y el elevado número de víctimas en un país que se consideraba bien preparado para los tsunamis sin duda creará grandes debates entre Ingenieros Costeros, las personas y organismos que gestionan zonas costeras y los gobiernos nacionales y locales. A pesar de las trágicas consecuencias de eventos como éste, es importante comprender que también presentan oportunidades para impulsar cambios en la utilización y protección de la costa e incrementar la capacidad de defensa de las comunidades contra futuros eventos. En este caso es difícil llegar a la conclusión de que los errores del pasado están siendo repetidos, debido a que este evento tiene un periodo de retorno tan alto que lo hace relativamente único en la historia de Japón hasta este momento. A pesar de esto, este tipo de eventos serán a partir de ahora una parte constituyente de la historia de la humanidad, y pueden servir de ejemplos para otras regiones de Japón o del mundo.

\section{Investigaciones en terreno}

Con el objetivo de obtener un registro completo de alturas de ola e impacto del tsunami en las regiones costeras, el Grupo de Investigación de Terreno del Tsunami fue organizado de manera inmediata después del evento. Este grupo estuvo compuesto de investigadores, docentes, ingenieros y funcionarios de diferentes organismos Japoneses. Tan solo dentro del Comité de Ingeniería de Costas Japonés fueron formados 48 equipos de trabajo, sumando en total más de 150 individuos. Además de esto, diversos otros equipos de trabajo fueron formados por autoridades de varias prefecturas y municipios, con lo cual es fácil concluir que se reunió más información acerca del tsunami que de cualquier otro en la historia. Gran parte de esta información es accesible en la página web del Tohoku Earthquake Tsunami Joint Survey Group (2011).

Los autores de este trabajo efectuaron cuatro investigaciones de terreno dentro de este grupo, en las prefecturas de Iwate, Miyagi, Fukushima, Ibaraki y Chiba. La altura de inundación debido al tsunami fue del orden de más de $10 \mathrm{~m}$ en la parte norte de Miyagi, 5 a $10 \mathrm{~m}$ en la Bahía de Sendai, y alrededor de $5 \mathrm{~m}$ en las costas de Ibaraki y Chiba. La Figura 1 muestra las zonas de la costa que fueron investigadas por los autores, indicando la altura de inundación en cada punto.

Es importante señalar que gran parte de la costa de Fukushima no fue inspeccionada debido a la zona de exclusión de $30 \mathrm{~km}$ impuesta por el gobierno japonés debido a la falla de la central nuclear en esta prefectura.
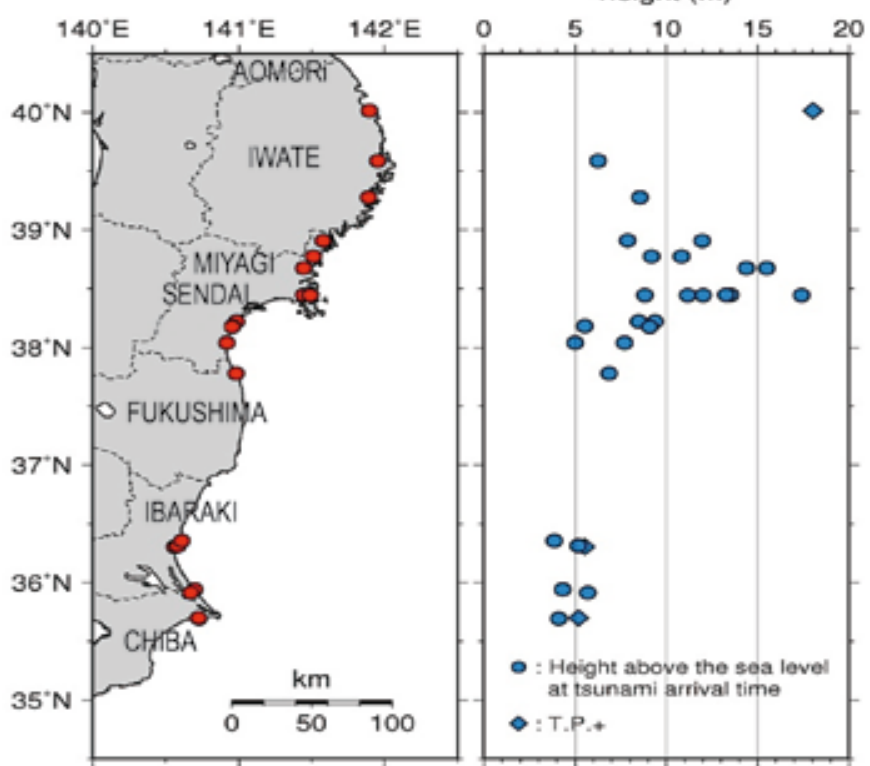

Figura 1: Altura de inundación del tsunami a lo largo de la costa japonesa

\section{Características geográficas del área de Tohoku}

El área costera de Tohoku es bastante variada desde el punto de vista de sus características geográficas, que deben ser consideradas detenidamente para comprender los daños 
causados por el tsunami en cada localidad. Una gran parte de la costa norte de Tohoku, y especialmente la Costa de Sanriku, está compuesta de rías (parecidos a los fiordos de Noruega pero que tienen su origen en la erosión causada por ríos en lugar de glaciares), mientras que la parte sur está caracterizada por la presencia de largas playas arenosas.

Las defensas costeras en la parte norte estaban generalmente compuestas por rompeolas, diseñadas para resistir tsunamis u olas generadas por viento. La parte sur en cambio disponía de menos rompeolas, y en lugar de ello las defensas costeras estaban compuestas de diques construidos para proteger la zona de los efectos de las olas generadas por vientos debidos a tifones y otras tormentas.

\section{Características sociales e industriales de la zona de Sanriku}

La parte norte de Tohoku desde un punto de vista socioeconómico está caracterizada por un descenso gradual de la población debido al envejecimiento provocado por bajas tasas de natalidad. La industria está dominada por pequeños puertos pesqueros y por el sector agrícola, con la excepción de algunas grandes ciudades como Miyako, Kamaishi, Ofunato y Kesennuma. Aunque estas ciudades también padecen un envejecimiento gradual de la población, el hecho de que algo de industria pesada y pesca moderna esté concentrada alrededor de estos puntos les convierte en pequeños centros regionales. La ciudad de Sendai es el núcleo urbano más importante de la zona de Tohoku, con una población de más de un millón de habitantes. Concentra las actividades comerciales de la zona y también es la capital de la región. Al sur de Sendai el sector agrícola es la principal actividad debido a que la zona está compuesta de zonas planas adecuadas al cultivo de arroz.

\section{Historia de los tsunamis y terremotos en el área de Tohoku}

A lo largo de los más de 1000 años de historia japonesa existen un gran número tsunamis detallados en las crónicas históricas del país. De acuerdo con estas crónicas en el área de Tohoku los 5 de mayor magnitud fueron los de Jogan (869), Keicho (1611), Meiji-Sanriku (1896), ShowaSanriku (1933) y Chile (1960) (Watanabe, 1985).
La descripción del tsunami de Jogan solo aparece en un documento histórico conocido como Sandai-Jitsuroku. Este tsunami inundó gran parte de la zona costera de Tohoku, y debido a ello perecieron aproximadamente unas 1000 personas. No existe ninguna otra fuente de información sobre este evento y por lo tanto el conocimiento que existe sobre el tsunami de Jogan es limitado. A pesar de ello, depósitos geológicos de este tsunami fueron encontrados recientemente en la llanura de Sendai y a lo largo de la costa de Sanriku, lo cual ha servido para identificar de forma gradual el área que fue inundada (Minoura et al., 2001). Desde la época Edo ( 1603), la cantidad de registros sobre los tsunamis se ha incrementado considerablemente. El tsunami de Keicho fue uno de los más destructivos de la época Edo, golpeando un área extensa que abarcó desde la isla de Hokkaido al norte hasta Sanriku. Desde el comienzo de la época de Meiji ( 1868) han tenido lugar tres grandes tsunamis en la costa de Sanriku. Dos de ellos son tsunamis que tuvieron su origen en terremotos cercanos a la costa mientras que el tercero tuvo su origen lejos de Japón. El primero de ellos se conoce como el tsunami de Meiji-Sanriku, que causó la pérdida de más de 22000 vidas. La magnitud del terremoto que originó el tsunami no fue demasiado grande, pero a pesar de ello las olas del tsunami llegaron a los $20 \mathrm{~m}$ de altura. El segundo tsunami se conoce como el tsunami de Showa-Sanriku, en el que perdieron la vida alrededor de 3000 personas. El tercer evento fue el tsunami de Chile de 1960, que fue producido por un terremoto de magnitud 9.5 en la escala Richter. Este tsunami causó graves daños a lo largo del Océano Pacífico, incluyendo la costa de Sanriku, donde murieron más de 100 personas.

\section{Daños estructurales}

Muchos edificios, inclusive edificios de hormigón armado, fueron arrasados por el tsunami, e incluso gran parte de los que permanecieron en su sitio sufrieron daños graves. El tsunami afectó de forma significativa a los rompeolas, diques, bosques y otras estructuras de defensa a lo largo de la costa. Es significativo que incluso rompeolas que fueron diseñados específicamente para proteger contra los tsunamis fueron total o parcialmente dañados. En esta sección se analizarán algunas características de los daños estructurales, las lecciones aprendidas a raíz de estos daños y de algunas áreas de investigación que deberían 


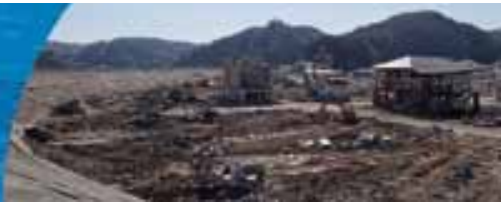

ser priorizadas en el futuro. En la opinión de los autores sería importante que en el futuro las estructuras costeras fueran diseñadas de un modo que no fallen de forma catastrófica incluso en el caso de que el criterio de diseño sea sobrepasado.

\section{Daño estructural a edíficios}

Un gran número de edificios fue parcial o completamente destruido y el daño que sufrieron las estructuras de madera no resulta sorprendente en este caso, pero muchos edificios de hormigón armado también sufrieron grandes daños. Particularmente elocuente es el caso de varios edificios en Onagawa, que sufrieron volcamiento producto del tsunami, como se puede ver en la Figura 2. Estos edificios fueron construidos sobre pilotes, y como consecuencia del terremoto fallaron por corte en la unión con los dados de fundación. Tras de ello, las fuerzas laterales debidas a la presión del agua volcaron la estructura. Es posible que la licuación del suelo de fundación pueda haber contribuido al colapso del edificio. Muchos de los edificios que sufrieron daños estructurales debido al tsunami fueron construidos hace varias décadas, usando códigos de diseño que no incluyen en su análisis la fuerza del flujo generado por un tsunami.

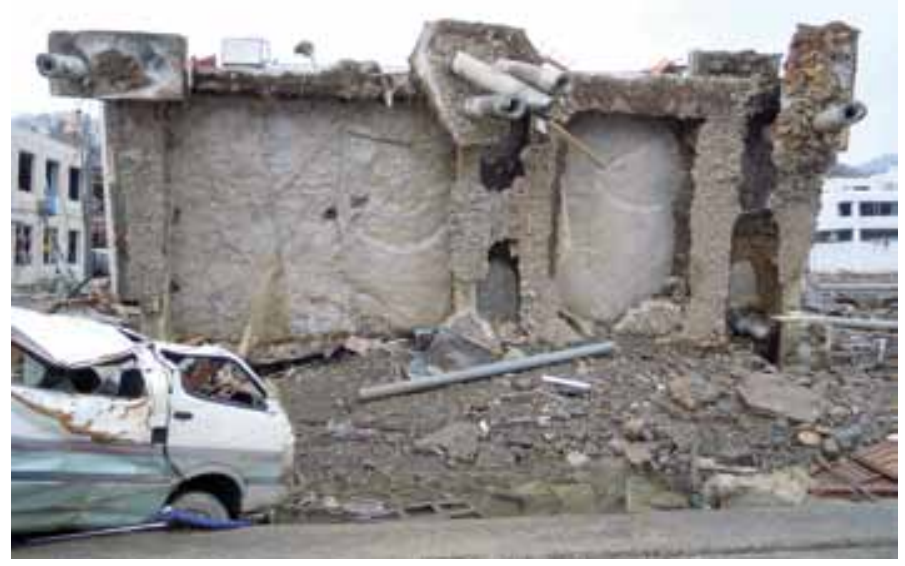

Figura 2: Edificio de hormigón armado fundado sobre pilotes volcado por el tsunami en Onagawa

En realidad, hoy en día sigue sin existir un código de diseño que haga obligatorio que las estructuras sean diseñadas para resistir un tsunami, ni en Japón ni en ninguna otra parte del mundo. El único documento disponible en Japón es el "Manual de Diseño de Edificios de Evacuación para
Tsunami”, que hasta ahora solo ha sido utilizado para el diseño de un número limitado de estructuras, como el edificio de evacuación de Minamisanriku (ver Figura 3) que sobrevivió el tsunami sin grandes daños estructurales, con la excepción de la socavación del terreno alrededor del edificio. A pesar de ello, este documento no tiene el estatus de un código de diseño obligatorio. Es interesante de todos modos observar que el nuevo código de diseño de edificios en Japón contiene información de cómo estimar las fuerzas laterales debido a un terremoto, y aunque éstas no tienen que ver directamente con un tsunami, un edificio diseñado con estas normas sería mucho más resistente a un tsunami que edificios construidos con códigos anteriores. En el caso de los Estados Unidos, algunos documentos contienen apartados que mencionan como calcular las fuerzas de un tsunami como el CCH (2000) o el FEMA P646 (2008), aunque ninguno de estos códigos tiene carácter obligatorio (Raskin et al. 2011).

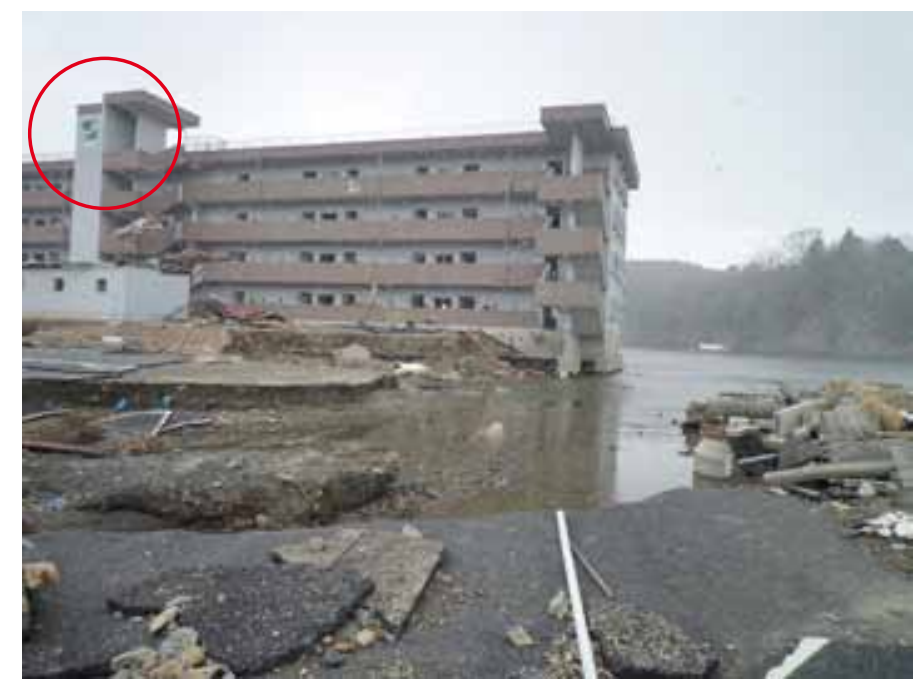

Figura 3: Edificio de evacuación (círculo rojo) de Minamisanriku con accesos socavados

Es interesante el hecho de que existen considerables diferencias entre los códigos de construcción y diseño existentes que disponen de apartados de cómo calcular las fuerzas debidas a un tsunami en función de la altura de inundación y de la pendiente de la playa. Algunos ejemplos de estos códigos son CCH (2000), ASCE 07 (2005), FEMA55 (2005) y FEMA P646 (2008). Investigadores como Nistor et al. (2009) han presentado nuevas fórmulas y metodologías para estimar las fuerzas debidas a un tsunami y cómo éstas actúan sobre edificios. A pesar de esto, 
existen considerables diferencias entre las estipulaciones y recomendaciones de los diferentes códigos: algunos de ellos, como el FEMA55 (2005), no consideran las fuerzas debidas al tsunami, o ciertos parámetros como la velocidad o las características del impacto de objetos flotantes son estimados de forma diferente en cada documento (Nistor et al. 2010). En realidad, la compleja interacción entre las fuerzas hidrodinámicas inducidas por el tsunami y las estructuras todavía no se comprende bien, aunque esta área está recibiendo bastante atención últimamente y se están realizando esfuerzos para mejorar los códigos de diseño. Un nuevo subcomité del ASCE7 ha sido formado con el objetivo de elaborar nuevas directrices para la estimación de fuerzas de los tsunamis, las cuales deberían ser publicadas para el año 2016. Al mismo tiempo en Japón se ha comenzado a trabajar para revisar las directrices de diseño de edificios situados en áreas de riesgo de tsunami.

\section{Daño estructural de diques costeros}

Numerosos diques costeros sufrieron considerables o irreparables daños debido al tsunami de marzo del 2011, que tuvo una altura de ola muy superior a la que se utilizó para diseñar estas estructuras, las cuales fueron diseñadas para olas de viento o de tsunami. Por supuesto que una estructura diseñada para olas de viento puede también ofrecer algo de protección contra un tsunami, no obstante, existen problemas particulares asociados a cada tipo de estructura. El área que concentra casi todas las estructuras de protección contra tsunamis en Japón es de hecho el área de Sanriku (Aomori, Iwate y Minyagi), debido a la frecuencia con la que los tsunamis han atacado esta costa en la historia reciente. En cambio, el área sur de Sanriku (en la llanura de Sendai) estaba protegida principalmente con estructuras diseñadas para proteger contra el aumento del nivel del mar durante temporales y tormentas.

Para el caso de diques diseñados para proteger contra olas generadas por el viento, la elevada fuerza causada por el caudal que sobrepasó la estructura resultó en la destrucción de muchas secciones de dique, como puede verse en la Figura 4. Muchas de estas estructuras estaban compuestas de un núcleo de gravilla y arena, recubierto de una estructura de hormigón armado con bajas cuantías de acero o sin refuerzo. Debido a la socavación progresiva, el núcleo de los diques quedó expuesto a la acción del agua lo que provocó su eventual colapso (ver Figura 5). Muchas de las partes centrales de los diques no estaban protegidas con geotextiles, y los autores observaron que los terraplenes en las riberas de los ríos (como en la desembocadura del río Natori) que sí estaban protegidas con geotextiles sufrieron menos socavación que en áreas que no lo estaban. Para mejorar la resistencia de este tipo de estructuras en futuros tsunamis se recomienda la posible inclusión de geotextiles en el diseño de estructuras de protección costera. De hecho, el uso de geotextiles es muy frecuente hoy en día en Japón, aunque se utilizan generalmente sólo en la parte frontal de las estructuras de protección, y teniendo en cuenta lo que sucedió en este último tsunami, quizás sería recomendable también instalarlas en la parte posterior.

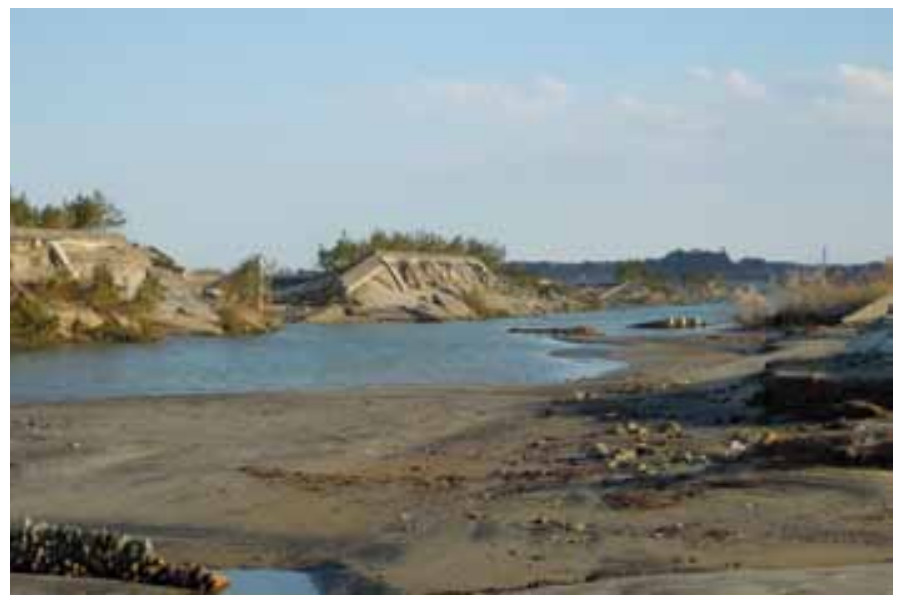

Figura 4: Colapso de una estructura de protección en la ciudad de Soma (Prefectura de Fukushima). Las partes de la estructura que sobrevivieron estaban reforzadas con tetrápodos colocados delante de ellos.

El efecto de los tetrápodos colocados enfrente de las estructuras de protección también merece la pena ser mencionado. En la ciudad de Soma estas unidades fueron capaces de proteger el núcleo del dique. Sin embargo, se debe mencionar que el uso de este tipo de estructuras, perjudica gravemente la estética de la playa. Por lo tanto, llegar a un equilibrio entre la necesidad de utilizar estructuras de protección y la preservación de la belleza del medio litoral, parece ser un problema no muy fácil de solucionar.

\section{Daño estructural a rompeolas}

Un gran número de rompeolas construidos en las áreas costeras de Tohoku pueden ser divididos en dos categorías, dependiendo de si fueron construidos principalmente para 
proteger contra olas de tsunamis o contra olas generadas por el viento.

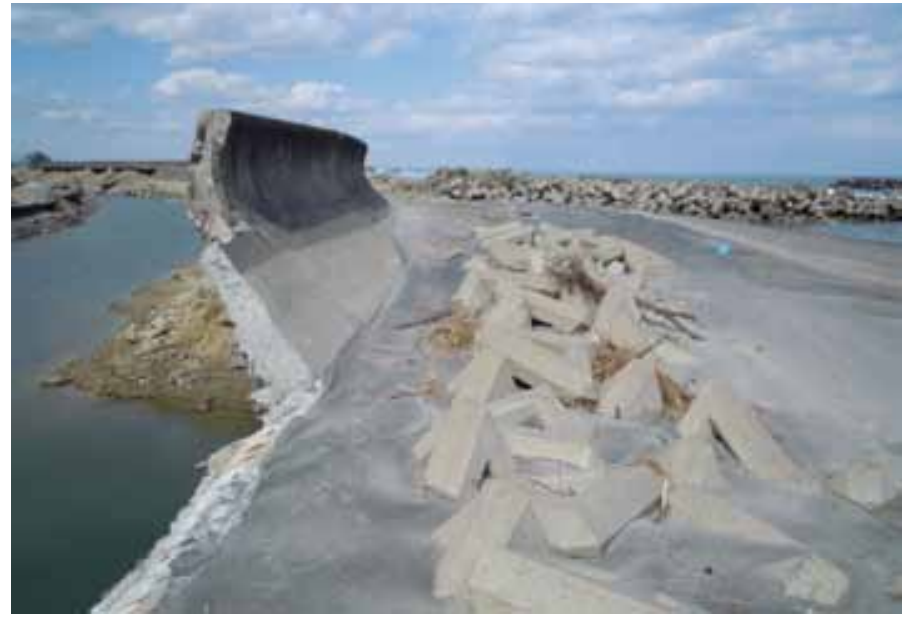

Figura 5: Ruptura de las estructuras de defensa en Watari. La socavación de la parte posterior del dique deja expuesto el núcleo de arena a la acción del agua, lo cual eventualmente también resultó en la rotura de la parte frontal del dique construida de hormigón.

Una gran parte del área costera de Tohoku, y especialmente el área de Sanriku, está formada por rías, antiguos valles fluviales inundados por el mar (parecidos a los fiordos) que son especialmente vulnerables a los tsunamis debido a que concentran gran parte de la energía del tsunami a lo largo de su eje longitudinal, tal y como ocurrió por ejemplo durante el tsunami de Chile en 1960. A raíz de este tsunami, el gobierno japonés decidió construir colosales rompeolas para proteger la entrada de algunas de las rías, en lugares como Kamaishi, Ofunato y Kuji. La construcción de dichas estructuras fue extremadamente costosa, debido a la elevada profundidad de la zona donde se encuentran situadas. De hecho el rompeolas de Kamaishi es el más profundo jamás construido en el mundo, a una profundidad de $63 \mathrm{~m}$. A pesar de ello estas estructuras no fueron capaces de sobrevivir intactas el Gran Terremoto y Tsunami del Este de marzo del 2011. El rompeolas de Ofunato desapareció completamente a causa del tsunami, y el de Kamaishi fue gravemente dañado. Se puede argumentar que en realidad ninguno de ellos fue diseñado para una altura de tsunami en mente igual a la que ocurrió durante el evento de marzo del 2011. Originalmente fueron concebidos para una altura de $6 \mathrm{~m}$ desde el nivel mínimo del mar a la parte superior del rompeolas (Takahashi et al. 2011). Es interesante de todos modos observar como los rompeolas de Kamaishi y
Ofunato se comportaron de forma diferente, el de Ofunato desapareció completamente (daño catastrófico) y el otro sufrió graves daños (en una de sus secciones al menos) pero permanece a la vista después del tsunami. Obviamente cada uno de los rompeolas tenía diferentes dimensiones y es normal también que reaccionaran de forma diferente. De todos modos, sería necesario investigar en el futuro de forma más detenida el mecanismo de falla de los rompeolas para tsunamis de gran altura. Es imperativo que en el futuro el diseño de rompeolas para tsunamis sea capaz de asegurar estructuras que no fallen de forma catastrófica.

No está claro tampoco cuánta protección ofrecieron estas estructuras a las poblaciones situadas detrás de ellas. Existe evidencia de que la altura de inundación detrás de las estructuras fue menor que delante de ellas (PARI, 2011). Aunque será necesario realizar estudios más detallados para modelar el comportamiento e interacción entre la ola y el rompeolas. También es importante señalar que actualmente no se comprende del todo el modo de destrucción de los rompeolas debido a un tsunami o incluso a las simples olas producidas por el viento (Esteban et al., 2008), y es evidente que esto tiene un efecto en la propagación de la ola dentro de la bahía.

Un elevado número de los rompeolas que fueron diseñados para proteger contra olas generadas por tormentas y tifones sufrieron también grandes daños, y en algunos casos fueron completamente destruidos, como el rompeolas a la entrada del puerto de Onagawa (ver Figura 6). Estos rompeolas, como resulta evidente, no fueron diseñados con tsunamis en mente y por tanto resulta lógico que fueran dañados.

\section{Efectos sobre el ambiente}

Tsunamis de la magnitud del Gran Terremoto y Tsunami del Este de marzo del 2011 tienen inevitablemente un efecto negativo sobre el ambiente litoral de las áreas afectadas. A continuación se presentan algunos de los efectos más inmediatos que se pueden observar en las costas de Tohoku.

\section{Erosión de las costas}

Los tsunamis pueden causar una importante erosión en las zonas costeras, debido al hecho de que pueden mover grandes cantidades de materiales de una zona a otra. Por lo tanto, algunas áreas pueden sufrir considerable erosión, 
a)

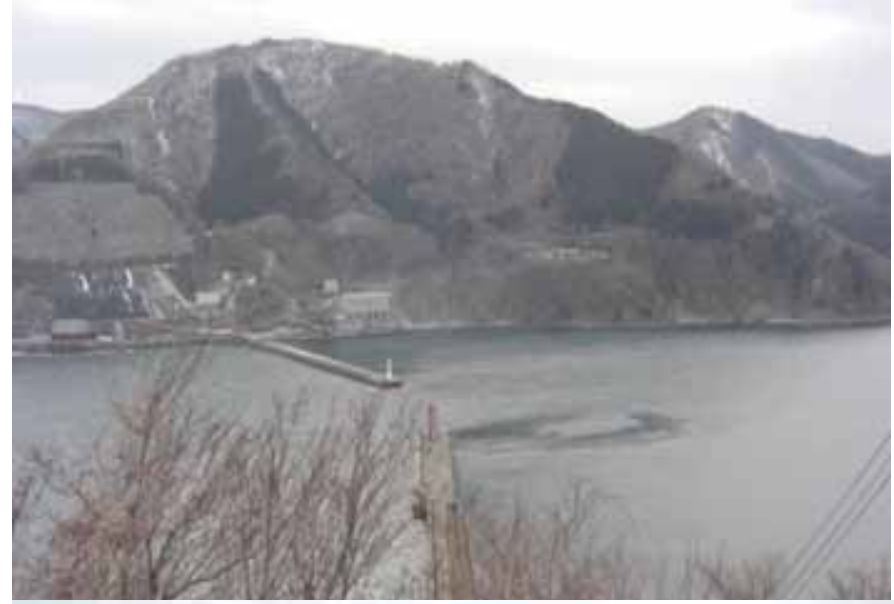

b)

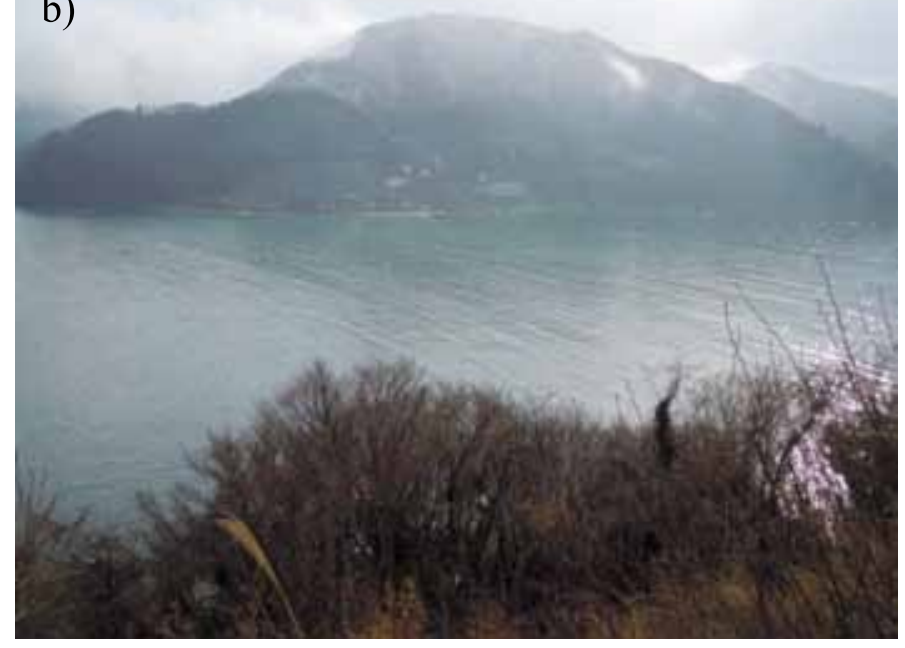

Figura 6: a) Rompeolas en Onagawa antes del tsunami y b) después del tsunami. Notar que prácticamente no hay vestigios del rompeolas

y luego estos sedimentos pueden estar depositados en otras zonas de la costa o tierra adentro. Para prevenir la erosión de estos y otros tipos de desastres costeros (como los causados por los tifones) las autoridades japonesas han construido en el pasado de forma sistemática estructuras de defensa costera a lo largo del litoral. A pesar de ello muchas, si no casi todas, fueron sobrepasadas por el tsunami de marzo del 2011, lo cual resultó en alteraciones dramáticas de la línea de costa. Ejemplos de esto pueden ser observados en partes como Minamisanriku o Motoyoshi, donde en algunos casos decenas de metros de litoral fueron perdidos. Recuperar estas zonas es por supuesto posible, pero conlleva mover grandes cantidades de materiales de unos sitios a otros para rellenar las zonas perdidas. Además, a consecuencia del terremoto y del movimiento que éste causó sobre la placa continental sobre la que se sitúa Japón toda el área de Tohoku ha perdido altura con respecto al nivel del mar, y esto ha agravado los efectos del tsunami. Algunos sitios como Ishinomaki han sufrido una subsidencia de $78 \mathrm{~cm}$ de acuerdo con la Autoridad de Información Geoespacial de Japón (GIAJ 2011), y en algunos lugares el desplazamiento vertical ha sido de hasta $1.2 \mathrm{~m}$. De forma adicional es posible que la erosión en la costa se incremente en el futuro debido al aumento de la profundidad del mar en estas zonas, que conllevará cambios en el régimen de olas de la zona. Es probable por lo tanto que los efectos del tsunami se continúen sintiendo todavía durante muchos años, y que considerables cantidades de dinero tendrán que ser gastadas para que la costa vuelva a la situación como estaba antes del tsunami. De forma general, es posible decir que las áreas que sufrieron menos erosión fueron las que estaban protegidas por estructuras que sobrevivieron el tsunami, lo cual señala la importancia de construirlas de forma adecuada.

\section{Bosques costeros y calidad del suelo}

Aunque existe una creencia generalizada de que los bosques costeros pueden ayudar a proteger contra un tsunami en realidad no está claro si en realidad estos ofrecen mucha protección. También es importante observar que los bosques pueden sufrir graves daños a consecuencia de un tsunami. Los tsunamis más fuertes son capaces de arrancar árboles y transportarlos al interior, convirtiéndolos en objetos flotantes que pueden incrementar el daño a las infraestructuras o edificaciones. Este proceso puede aumentar el daño de la zona, como aconteció en Rikuzentakata, donde el bosque costero fue completamente arrasado por el tsunami, que causó también una importante erosión en el área costera circundante (ver Figura 7).

Los tsunamis también pueden incrementar la salinidad de los suelos de las áreas inundadas, lo cual puede resultar en la muerte de la vegetación, como fue observado en numerosas áreas donde las hojas de los árboles gradualmente cambiaron de color después del tsunami. Esto indica el efecto causado por el incremento de la salinidad, aunque por supuesto es posible que la vegetación se recupere. De 
todos modos es importante recordar que este efecto no debería distraer la atención de problemas más importantes como la preservación de las vidas humanas, mucho más importante que el estado de la vegetación después de un tsunami.

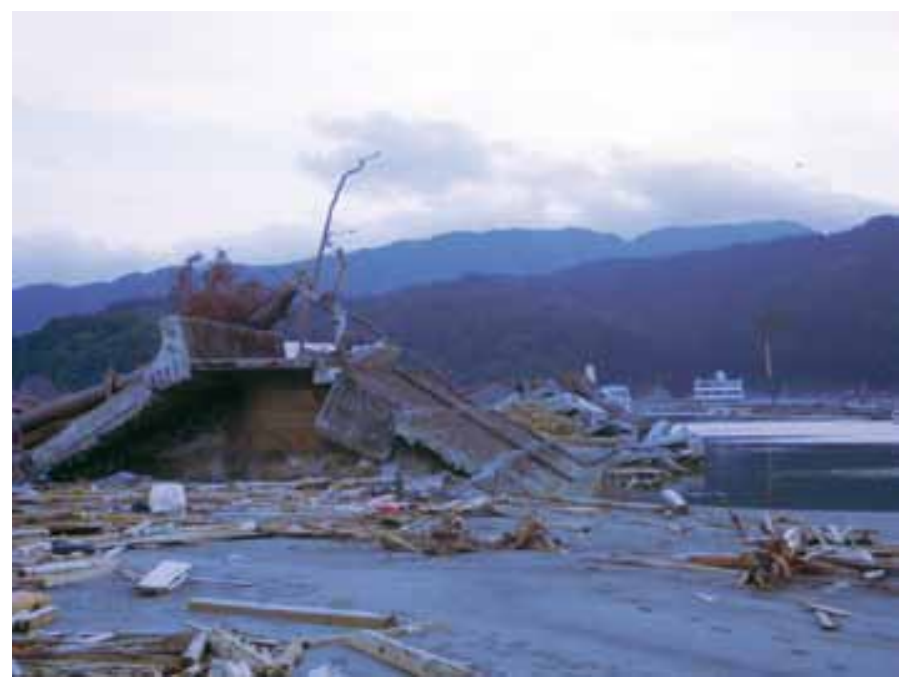

Figura 7: Solo un árbol del bosque costero de Rikuzentakata sobrevivió al tsunami

\section{Consideraciones relacionadas con las pérdidas humanas}

El Gran Terremoto y Tsunami del Este de marzo del 2011 causó más de 20000 muertes, número elevado teniendo en cuenta que Japón era un país que se consideraba especialmente bien preparado para resistir desastres naturales. Anteriores desastres como el tsunami de Meiji-Sanriku en 1896 (21959 víctimas entre muertos y desaparecidos), el tsunami Showa-Sanriku en 1933 (1522 muertos y 1542 desaparecidos) muestran como el área ha sufrido y probablemente continuará sufriendo devastadores tsunamis. En términos de magnitud, parece que el tsunami de Meiji-Sanriku en 1896 causó olas que llegaron a los $38.2 \mathrm{~m}$ de altura en Ryori, con lo cual sería comparable con el evento del 2011. Resulta por tanto importante intentar comprender por qué murieron tantas personas, y qué lecciones se pueden sacar para mejorar la preparación con respecto a futuros tsunamis.

\section{Refugios para tsunami y edificios de evacuación}

Uno de los pilares de la filosofía de prevención de desastres radica en la necesidad de entrenar a la población local a que evacue el área y se dirija a zonas elevadas tras la emisión de una alerta de tsunami. Cuando estas zonas elevadas no son fácilmente alcanzables, la población debería dirigirse a estructuras elevadas, que pueden haber sido construidas para el solo propósito de ofrecer refugio contra tsunamis, a los que nos referiremos como "Refugios para tsunami", o edificios altos y robustos que generalmente se dedican a otra función como hospitales o bloques de viviendas, a los cuales nos referiremos como "Edificios de Evacuación". La distinción entre estos dos tipos de edificios es importante dentro del contexto de la terminología de Gestión de Desastres Naturales en Japón, y por tanto mantendremos esta terminología en este artículo.

Los Refugios para tsunami y Edificios de Evacuación generalmente son diseñados teniendo en cuenta el peor escenario que se puede elaborar usando la información científica e histórica disponible. Sin embargo, en el caso del Gran Terremoto y Tsunami del Este de marzo del 2011, algunas de las construcciones que habían sido diseñadas como Edificios de Evacuación eran de menor dimensión que la altura del tsunami. Un ejemplo de esto ocurrió en Minamisanriku, donde la persona que emitió la alarma del tsunami permaneció en su puesto hasta que la ola destruyó el edificio. Durante una de las investigaciones de terreno llevadas a cabo por los autores, uno de los residentes del Edificio de Evacuación que se puede ver en la Figura 3 explicó cómo estando en la azotea del edificio tuvo que levantar a su hijo en brazos para que la ola no le mojara. Este edificio tenía 4 plantas, pero algunos otros tenían menos altura y cualquiera que se refugiara en ellos probablemente pereció como consecuencia de que la ola lo rebasara.

Debido a todo esto es importante que se revise la filosofía utilizada para el diseño de Edificios de Evacuación. La construcción de estructuras altas y robustas que puedan ser utilizados como Edificios de Evacuación es claramente posible desde un punto de vista técnico y económico en un país como Japón. Estos edificios, si están bien diseñados, parecen ser capaces de resistir las fuerzas del tsunami, como se muestra en la Figura 3, aunque es necesario de todos modos continuar investigando como mejorar el tipo de construcciones. En el futuro, los Edificios de Evacuación deberían ser diseñados teniendo en mente el tsunami más alto que se espere en un determinado periodo de tiempo, independientemente de que existan registros 
históricos o no sobre ello. Cómo seleccionar este periodo de retorno no está claro, pero el Gran Terremoto y Tsunami del Este claramente crea un precedente para intentar construir Edificios de Evacuación contra el tsunami más alto que haya acontecido desde un punto de vista geológico e histórico. Existen, por supuesto, limitaciones al tipo de tsunami contra el que se puede intentar diseñar, y para el caso de eventos extremadamente raros, como impactos de meteoritos que pueden generar olas de varios cientos de metros de altura, los métodos tradicionales de gestión de desastres naturales de la Ingeniería de Costas son probablemente inefectivos. Estos eventos se podrían encuadrar dentro de otro nivel diferente de tsunamis, que probablemente se podría denominar como "Nivel 3". La gestión de desastres de tsunamis provocados por eventos tipo meteoritos probablemente debería utilizar tecnologías de satélites y telescopios para predecirlos con días de anterioridad e intentar evacuar a toda la gente de áreas costeras. En este artículo no discutiremos más sobre este tipo de mega-tsunamis, debido a que están fuera del área de estudio típica de los Ingenieros de Costas.

En la opinión de los autores este cambio de filosofía de intentar proteger contra eventos de mayor magnitud requerirá una reevaluación de los riesgos a lo largo de las diferentes secciones de la costa. Por fortuna para el caso de Japón existen un gran número de universidades y centros de investigación sobre tsunamis, por lo que ya existe un abundante número de personas formadas sobre el tema. Además de esta reevaluación será necesario seleccionar la forma de evacuar de acuerdo con las características topográficas del área, por lo que lugares como Sanriku y Natori deberían utilizar diferentes estrategias para proteger a sus poblaciones.

Los autores de este artículo argumentan que los Puntos de Evacuación en Japón deberían ser clasificados utilizando tres categorías diferentes:

- Categoría A. Esta categoría incluiría colinas (u otras zonas elevadas) que se sitúen cerca de la costa pero que continúen elevados durante bastante distancia. De preferencia no serían colinas aisladas pero parte de sistemas montañosos más extensos. Un buen ejemplo de esto sería Akanumayama, cercana a Taro, que está ya designada como Punto de Evacuación.

- Categoría B. Que incluiría edificios robustos de más de 6 pisos, o colinas aisladas de más de $20 \mathrm{~m}$ de altura. Esta categoría tiene el riesgo inherente de poder quedarse aislada en el caso de los peores tsunamis, pero debería ser segura para la mayor parte de los eventos. Todos los nuevos Edificios de Evacuación construidos a partir de ahora deberían ser por lo menos de Categoría B.

- Categoría C. Incluiría edificios robustos de más de 4 pisos. Esta categoría, sin embargo, corre el riesgo de que en el caso de los peores tsunamis el edificio podría ser completamente rebasado por la inundación. El uso de edificios de esta categoría no estaría recomendado, pero en áreas donde no existan edificios de Categoría A o B se podrían utilizar durante el tiempo que mejores Edificios de Evacuación no sean construidos. Ningún Edificio de Evacuación nuevo debería ser construido en esta categoría.

Existiría por lo tanto una jerarquía de Puntos de Evacuación, con lo cual la población local debería dirigirse siempre a los de Categoría A, si estos no están disponibles o se encuentran muy lejos a los de Categoría B, y solo dirigirse a los de Categoría $\mathrm{C}$ como último recurso. En estos momentos el Profesor Shibayama está dialogando con las autoridades de la Prefectura de Kanagawa y la ciudad de Yokohama para la implementación de un sistema de evacuación basado en estas ideas. Sin embargo, en algunas áreas no existen siquiera Edificios de Evacuación de Categoría $\mathrm{C}$, por ejemplo en áreas portuarias con una gran concentración de almacenes de baja altura, en los que habitualmente hay muchos trabajadores durante el día pero que están prácticamente desiertos por la noche. Esto presenta un problema significativo, y cómo proteger a los trabajadores de estas áreas tiene que ser considerado de forma cuidadosa.

\section{Defensas costeras}

Es comprensible que los residentes de un área en la que existe peligro de tsunami puedan sentirse protegidos gracias a las defensas costeras construidas en estas áreas (ver Figura 8), lo cual puede haber contribuido al elevado número de víctimas. Sin embargo, pese a que estas estructuras pueden ser efectivas contra eventos de menor magnitud, en el caso del Gran Terremoto y Tsunami del Este de marzo del 2011 no fueron suficientes para contener 
la inundación, resultando en la destrucción del área detrás de ellas. En el caso de Taro (Figura 8) está claro que aunque las defensas resistieron bien el ataque, los edificios situados detrás de ellas fueron altamente vulnerables a la ola una vez que ésta rebasó las defensas.

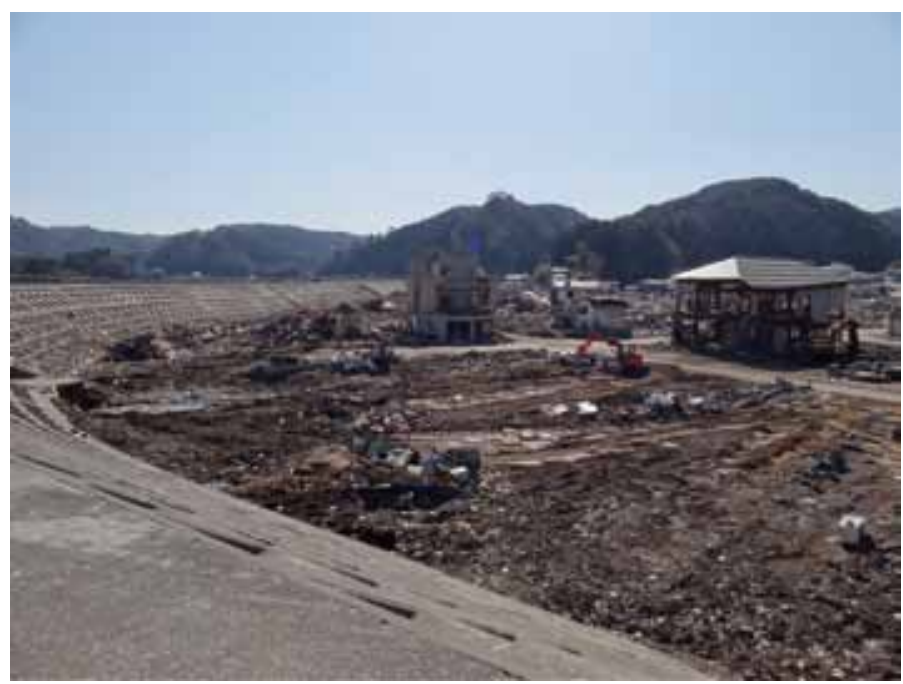

Figura 8: Devastación detrás de las defensas costeras de Taro

Es difícil comprender realmente hasta que punto existía un exceso de confianza, ya que los individuos que no evacuaron el área en el tiempo disponible murieron. Los sobrevivientes, sin embargo, generalmente explican que eran conscientes de que las defensas anti-tsunami podrían ser rebasadas y que era necesario evacuar el área. De acuerdo a sus testimonios la mayor parte de las víctimas simplemente no tuvieron tiempo suficiente de evacuar antes de ser alcanzados por el tsunami.

Por lo tanto, una de las lecciones que se puede obtener de este evento es lo importante de enfatizar a la población local que deben siempre evacuar lo más rápidamente posible, independientemente de si existen estructuras de protección o no. La gente debería estar consciente de que la función principal de estas estructuras es la protección de los bienes materiales, y que éstas ofrecen poca o ninguna protección contra los tsunamis de mayor magnitud.

\section{Falta de líneas de visión}

En algunos lugares como Rikuzentakata hubo gente que no evacuó debido a que su línea de visión al mar estaba bloqueada por un bosque costero, con lo cual no fueron capaces de ver el tsunami a medida que se acercaba.
Estructuras masivas como la de Taro (Figura 8) pueden impedir a la población obtener señales visuales de que se acerca un tsunami, o incluso tapar parte del estruendo que suele causar este tipo de eventos. Esto indica cómo se debería intentar que la gestión de desastres naturales no se apoye únicamente sobre medidas de infraestructura o de planificación, y como unas pueden impedir que las otras funcionen de forma efectiva. Las medidas físicas pueden disminuir la cantidad de estímulos visuales o sonoros, y por lo tanto dar a los residentes una falsa sensación de seguridad.

\section{Futuras líneas de investigación}

A raíz de los serios daños que tuvieron lugar es aconsejable que la investigación sobre tsunamis se intensifique en el futuro. Antes del tsunami del Océano Indico del 2004 se realizaba relativamente poca investigación sobre tsunamis, y aunque después de este evento la investigación se intensificó en diferentes lugares del mundo, el grado de interés comenzó a decaer debido a que los tsunamis que le siguieron causaron solo daños locales en lugar de a una escala internacional (como por ejemplo los tsunamis de Chile e Indonesia en el 2010). Es obvio que se desconoce aún mucho sobre los tsunamis, y es importante que se continúe investigando sobre la generación, propagación, transformación e impacto de los tsunamis.

\section{Investigación sobre Paleotsunamis}

Uno de los mayores problemas para la gestión de riesgos relacionados con los tsunamis es que se utilizan los escritos históricos para analizar las amenazas futuras. La historia de la humanidad, sin embargo, solo tiene algunos miles de años en el mejor de los casos, y aunque algunos países disponen de documentos históricos de larga duración, estos raramente proporcionan mucha información sobre la magnitud de los eventos. En cualquier caso es necesario tener en cuenta eventos pasados, y para hacerlo los escritos históricos no siempre son suficiente. Un área de investigación relativamente reciente pero que ha ido ganando más atención es la de los paleotsunamis, en la cual eventos que son anteriores al comienzo de la historia humana (o que no están mencionados en la historia) son confirmados mediante la utilización de depósitos geológicos. Esta área es de una gran importancia para intentar establecer con qué frecuencia se puede esperar 
diferentes tipos de eventos en el futuro. Además, es necesario para los ingenieros comprender cuál es el peor evento posible que se puede esperar en un determinado número de años, a fin de poder diseñar estructuras de protección de forma adecuada. Aunque es imposible diseñar contra todos los eventos posibles, el conocer qué eventos sucedieron en el pasado indudablemente ayuda en la elaboración de estrategias de gestión de riesgo más apropiadas.

\section{Investigación sobre daño estructural y propagación}

Hoy en día la estimación de las fuerzas ejercidas por los tsunamis sobre las estructuras costeras y edificios no está todavía del todo elucidada, como fue explicado anteriormente. El modelamiento de estos eventos en el laboratorio no siempre se puede realizar de forma satisfactoria, a pesar de la existencia de algunos tanques de olas de grandes dimensiones como el situado en PARI, Yokosuka en Japón. Existen todavía muchos debates acerca de hasta qué punto es posible reproducir los tsunamis en el laboratorio, y si estos modelos son capaces de indicar de forma adecuada las fuerzas asociadas con estos eventos. Es imprescindible que se llegue a una mejor comprensión sobre las olas del tsunami para poder diseñar de forma más adecuada las estructuras situadas en áreas de alto riesgo, y esta área de investigación debería ser priorizada en el futuro. A medida que la población y la economía de diversos países continúan desarrollándose, es probable que el número de edificios en áreas de alto riesgo, como Indonesia por ejemplo, continúe incrementándose, y es importante que estas estructuras estén diseñadas de forma adecuada.

Para la simulación de tsunamis también se utilizan modelos numéricos, pero los resultados de la propagación de los tsunamis a veces tienen errores en los cuales la altura de la ola puede ser infra o sobre-estimada por un factor de dos o más. A menudo solo se puede estimar de forma adecuada la primera ola, debido a problemas de reflejo o el desconocimiento de los datos precisos sobre los parámetros de generación del tsunami, que dependen del movimiento original de la falla tectónica. Como ésta ocurre debajo del agua a menudo es difícil conocer los parámetros reales, lo cual dificulta también la estimación de la altura del tsunami.
Para poder formular escenarios adecuados para la gestión de riesgo de un tsunami en un área determinada es extremadamente importante poder predecir de forma espacial y temporal el impacto del tsunami. Es importante por lo tanto seguir mejorando las herramientas disponibles en estas áreas, que en estos momentos no son del todo adecuadas.

\section{Comunicación de riesgos y falta de coordinación entre investigadores y funcionarios del gobierno}

A menudo existe una falta de cooperación y comunicación entre investigadores de diferentes disciplinas, lo cual puede crear obstáculos para el desarrollo de herramientas adecuadas para el análisis de tsunamis. Los sismólogos a menudo están centrados sólo en los terremotos y no en los tsunamis que pueden ser generados como consecuencia de estos. Los investigadores de tsunamis a menudo utilizan parámetros obtenidos de los sismólogos, pero generalmente no se preocupan mucho de cómo fueron obtenidos estos datos o cuan fiables son. Los funcionarios del gobierno que están encargados de los planes de evacuación utilizan los resultados que les dan los sismólogos e investigadores de tsunamis, sin realmente saber de dónde proceden los resultados. Cada una de estas tres comunidades suele actuar de manera relativamente aislada a las otras, y sería aconsejable que se incrementara la cooperación y comunicación de riesgos entre ellas para mejorar la preparación contra desastres naturales.

\section{Comentarios finales y conclusiones}

En este artículo los autores intentan exponer cómo poner demasiado énfasis sólo en métodos de planificación o sólo en los métodos de infraestructura puede constituir un problema, y como en algunos casos un método puede interferir con el correcto funcionamiento del otro. Los métodos físicos pueden reducir la cantidad de tiempo de aviso que los individuos reciben y dar a los residentes una falsa sensación de seguridad. Los métodos de planificación, como los planes de evacuación, requieren que la población se mueva a zonas elevadas, y si éstas están muy lejos, a Refugios para tsunami o Edificios de Evacuación, que deberían ser diseñados de forma correcta. Una de las principales lecciones obtenidas de este desastre es que es necesario incrementar el entrenamiento de los residentes y hacerles conscientes de cómo los métodos físicos y de 
evacuación interactúan y se complementan. El uso de métodos físicos sigue requiriendo la evacuación del lugar, debido a que el tsunami puede ser mayor de lo que las defensas pueden resistir. Si la infraestructura se encuentra diseñada de forma adecuada, puede proporcionar a los residentes un tiempo valioso para evacuar el área. Sin embargo, el consenso general en Japón en estos momentos es que los métodos físicos no pueden proteger las vidas humanas en el caso de eventos de mayor magnitud. Estos métodos físicos pueden proteger los bienes materiales en el caso de los eventos de mayor frecuencia (pero menor magnitud), y puede que tengan también una función de protección de vidas en estos eventos menores. Este último punto aún constituye un tema de discusión entre funcionarios de gobierno e Ingenieros de Costas. La protección de las vidas humanas, sin embargo, siempre debería en última instancia basarse en métodos de planificación, y la población siempre debería evacuar las zonas costeras después de la emisión de una alarma de tsunami. El Gran Terremoto y Tsunami del Este de marzo del 2011 representa de forma obvia un evento de elevada magnitud, y la población debería estar entrenada para protegerse contra tales eventos.

Es necesario también establecer una jerarquía de Puntos de Evacuación que dé a la población indicación de una zona con los sitios más seguros a los cuales dirigirse. En el caso de una emergencia la población debería dirigirse a los puntos más seguros, y solo si juzgaran que no disponen de tiempo suficiente para llegar a ellos deberían dirigirse a Puntos de Evacuación considerados como menos seguros. Existe la posibilidad de que en el futuro las zonas costeras exhiban una diferenciación más radical de cómo utilizar cada tipo de terreno. Algunos investigadores y funcionarios del gobierno argumentan que varios pequeños poblados de pescadores podrían ser combinados en uno, donde las casas de los habitantes estarían situadas en zonas elevadas y solo el puerto se encontraría cerca del mar. Aunque esto suceda se debe considerar también de forma cuidadosa la evacuación de los trabajadores del puerto a zonas elevadas. Es necesario incrementar la cooperación y comunicación de riesgo entre las diferentes disciplinas académicas y los funcionarios de gobierno. Los expertos en desastres naturales deberían estar conscientes de las limitaciones de su investigación y de las medidas que proponen e implementan. Si se considera lo que sucede cuando una estructura resulta destruida, o las limitaciones de los métodos físicos y de planificación, es posible formular diferentes escenarios de gestión de riesgos. Es probable que si se hubiese analizado una serie de escenarios para las áreas afectadas que incorporaran las limitaciones de cada estructura, el daño podría haber sido reducido. En el futuro la comunicación entre la población local y los funcionarios de gobierno e ingenieros debería ser promovida para mejorar la preparación contra desastres naturales.

Otro problema previsible es que funciones de emergencia y rescate puedan fallar durante eventos de gran magnitud debido a diferentes problemas como apagones de electricidad, incremento del tráfico durante la evacuación, o incluso una falta de conocimiento de la población acerca de la alarma. Esto indica como los sistemas en los que se ancla la gestión de desastres naturales realmente no recibe la atención debida por parte de la sociedad en general. Para prevenir daños similares en otras áreas, un sistema comprensivo de respaldo debería ser instalado que incluya la posible falla de estructuras, sistemas de información, sistemas de evacuación, etc. Resulta imperativo que incluso en el caso de que un sistema falle los otros sigan funcionando.

\section{Referencias}

ASCE (2005). Minimum Design Loads for Buildings and Other Structures. ASCE/SEI Standard 7-05, Reston, Virginia, USA

City and County of Honolulu Building Code CCH (2000). Department of Planning and Permitting of Honolulu Hawaii. Chapter 16, Article 11. Honolulu, Hawaii.

Esteban, M., Nguyen, T., Takagi, H. and Shibayama, T. (2008). Analysis of rubble mound foundation failure of a caisson breakwater subjected to tsunami attack. $18^{\text {th }}$ International Offshore and Polar Engineering Conference, Vancouver

Federal Emergency Management Agency FEMA 55 (2000). Coastal Construction Manual.

Federal Emergency Management Agency FEMA P646 (2008). Guidelines for Design of Structures for Vertical Evacuation from Tsunamis. Washington, DC., USA

Geospatial Information Authority of Japan GIAJ (2011). Survey results of land subsidence due to the 2011 off the Pacific coast 
of Tohoku earthquake. Press release document, http://www.gsi. go.jp/sokuchikijun/sokuchikijun60008.html (en Japonés)

Minoura, K., Imamura, F., Sugawara, D., Kono, Y. and Iwashita, T. (2001). The 869 Jogan tsunami deposit and recurrence interval of large-scale tsunami on the Pacific coast of northeast Japan. Journal of Natural Disaster Science 23, 83-88

Nistor, I., Palermo, D., Nouri, Y., Murty, T. and Saatcioglu, M. (2009). Tsunami forces on structures. Chapter 11 in "Handbook of Coastal and Ocean Engineering”, World Scientific, Singapore, 261-286

Nistor, I., Palermo, D., Cornett, A. and Al-Faesly, T. (2010). Experimental and numerical modeling of tsunami loading on structures. International Conference of Coastal Engineering, ICCE2010, ASCE, Shanghai, China

Port and Airport Research Institute PARI (2011). Verification of breakwater effects in Kamaishi Ports. http://www.pari.go.jp/ info/tohoku-eq/20110401.html Accessed 19th July 2011 (en Japonés)
Raskin (2001). An evacuation building project for Cascadia earthquakes and tsunamis. Obras y Proyectos 9, 11-22

Sawai, Y., Okamura, Y., Shishikura, M., Matsuura, T., Than Tin, Aung, Komatsubara, J. and Fujii, Y. (2006). Historical tsunamis recorded in deposits beneath Sendai Plain -inundation areas of the A.D. 1611 Keicho and the A.D. 869 Jogan tsunamis. Chishitsu News No. 624, 36 -41 (en Japonés)

Takahashi, S., Kuriyama, Y., Tomita, T., Kawai, Y., Arikawa, T. Tatsumi, D. and Negi, T. (2011). Urgent Survey for 2011 Great East Japan Earthquake and Tsunami Disaster in Ports and Coasts. Technical Note of the Port and Airport Research Institute, No.1231, p.157 (en Japonés)

The 2011 Tohoku Earthquake Tsunami Joint Survey Group (2011). http://www.coastal.jp/ttjt/ Accessed 19 ${ }^{\text {th }}$ July 2011

Watanabe, H. (1985). Comprehensive bibliography on tsunami of Japan. University of Tokyo Press, Tokyo (en Japonés) 